\title{
Exploring the central kiloparsec in Seyfert galaxies
}

\author{
M. Salvato ${ }^{1}$, J. Greiner ${ }^{1}$ and B. Kuhlbrodt ${ }^{2}$ \\ ${ }^{1} \mathrm{MPE}$, Giessenbachstr. 1, 85748, Garching, Germany \\ email: mara@mpe.mpg.de, jcg@mpe.mpg.de \\ ${ }^{2}$ AIP, An der Sternwarte 16, 14482, Potsdam, Germany email: bkuhlbrodt@aip.de
}

\begin{abstract}
We collected X-ray and optical images for a X-ray flux-limited, complete sample of 93 active galactic nuclei (AGNs) at $z<0.1$, selected from the ROSAT Bright Survey. By modeling the host galaxy and the central AGN, we decompose the optical emission into nuclear, bulge and disk components. We cross-correlated our sample with the NVSS and FIRST radio catalogs, and, due to their limited sky coverage, 53 matches were found. We find that the nuclear optical luminosity, thought to be associated with the accretion disk surrounding the active black hole, correlates with the X-ray luminosity, the radio luminosity, and the black hole mass.
\end{abstract}

\section{Introduction}

For AGN with typical BH masses of $10^{6}-10^{9} \mathrm{M}_{\odot}$, the bulk of the accretion radiation is emitted in the soft X-ray and EUV/UV range. A fraction of this optically thick radiation is Compton-upscattered by a corona of electrons surrounding the BH. This process is thought to produce the typical power law X-ray spectra observed in all AGN. The primary soft X-ray/EUV radiation of the accretion disk also ionizes the surrounding gas, producing the well-known AGN emission lines. At radio frequencies, the core luminosity of the AGN is due to synchrotron emission by relativistic electrons immersed in a seemingly global magnetic field. It is conceivable that these electrons are the same which Compton-upscatter the disk photons. It is therefore interesting to ask whether there is a direct correlation between radio, optical and X-ray emission in the central part of the AGN where the accretion disk is located.

\section{Sample}

We analyzed an X-ray flux-limited (count rate $>0.3$ counts $\mathrm{s}^{-1}$ ), complete sample of 93 local $(0.009<z<0.1)$ AGN selected from the ROSAT Bright Survey (Schwope et al. 2000). Seyfert 1 and NLSy1 represent $2 / 3$ and $1 / 3$ of the sample, respectively. We used Optical data: we applied a 2-dimensional decomposition algorithm (Kuhlbrodt et al. 2004) on calibrated images, which gives as output the nuclear luminosity, together with the luminosities, PA and eccentricities of the spheroidal and the disk components.

Black hole masses: computed from the derived spheroidal luminosity using an unique equation for Sy1 and NLSy1 (McLure \& Dunlop 2002).

X-ray data: we computed X-ray nuclear luminosities using the flux measured by ROSAT in the $0.5-2 \mathrm{keV}$ range. We assumed a power law spectrum with a photon index of -2 and applying Galactic absorption and k-correction.

Radio data: We cross-correlated our sample with the NVSS (Condon et al. 1998) and FIRST (Becker et al. 1995) radio catalogs. Both surveys are centered at $1.49 \mathrm{GHz}(\lambda=$ $20 \mathrm{~cm}$ ), where the synchrotron emission of our targets may be associated with the nuclear 
activity but also with the spatially-extended star formation activity, if any. NVSS and FIRST map the sky at different spatial resolution (45 and 5 arcseconds, respectively), and thus a comparison of the fluxes in the two catalogs allows to disentangle the two potential contributions. For 21 galaxies the radio luminosity has been attributed only to their nuclear activity.

\section{Results}

We find correlations between $i$ ) the optical luminosity of the nuclei of Seyfert galaxies, ii) the power-law X-ray emission and iii) the radio emission from the core (Eq. 1 and 2). Moreover, the optical luminosity correlates also with the BH mass in such a way that for a given $\mathrm{BH}$ mass the optical flux depends on the accretion rate (see for details Salvato et al. 2004). These relations are not driven by the Malmquist bias, which is introduced whenever a sample is selected via a flux-limit.

$$
\begin{gathered}
\log \left(L_{X}\right)=0.58 \log \left(L_{R(N u c)}\right)+18.44 \\
\log \left(L_{F I R S T(N u c)}\right)=0.96 \log \left(L_{X}\right)-4.18
\end{gathered}
$$

The relations are also confirmed by the Spearman Rank correlation coefficient, which gives in both cases a probability lower than $0.1 \%$ that the relations are introduced by the common dependence of the variables on the distance.

\section{Conclusions}

The above correlations between the nuclear radio, X-ray and optical luminosity may have their origin in the electron corona surrounding the accretion disk. The electron temperature depends primarily on the accretion rate and disk temperature. Moreover, the temperature and density of the corona determine the efficiency of the Compton scattering, and thus directly the slope and power of the X-ray spectrum. A change in the accretion rate would not only lead to a varying optical emission, but via the corona also to a varying X-ray and possibly radio emission. In microquasars, Rau \& Greiner (2003) have found a similar correlation between the strength of the radio emission and the X-ray power law slope. Adopting this similarity, one could argue that the core radio emission in Seyfert galaxies traces matter outflow from the central black hole. Objects with higher activity at X-ray wavelengths, i.e. higher accretion rate, therefore exhibit higher mass outflow from their central engine, thus implying a larger radio flux. Support to this idea also comes from BH binaries studies (Gallo et al. 2003) and super-massive BHs (Merloni et al. 2003).

\section{References}

Becker, R. H., White, R. L., \& Helfand, D. J. 1995, ApJ, 450, 559

Condon, J. J., et al. 1998, AJ, 115, 1693

Gallo E., Fender, R. P., \& Pooley, G. G. 2003, MNRAS, 344, 60

Kuhlbrodt, B., Wisotzki, L., \& Jahnke, K. 2004, MNRAS, 349, 1027

McLure, R. J., \& Dunlop, J. S. 2002, MNRAS, 331, 795

Merloni, A., Heins, S., \& di Matteo, T. 2003, MNRAS, 345, 1057

Rau, A., \& Greiner, J. 2003, A\&A, 397, 711

Salvato, M., Greiner, J., \& Kuhlbrodt, B. 2004, ApJ, 600, L31

Schwope, A., et al. 2000, Astron. Nach., 321, 1 